\title{
Neuromyelitis optica spectrum disorders
}

\author{
Authors: Saif Huda, ${ }^{\mathrm{A}}$ Dan Whittam, ${ }^{\mathrm{B}}$ Maneesh Bhojak, ${ }^{\mathrm{C}}$ Jayne Chamberlain, ${ }^{\mathrm{D}}$ Carmel Noonan, ${ }^{\mathrm{E}}$ Anu Jacob ${ }^{\mathrm{F}, \mathrm{G}}$ and \\ Rachel Kneen ${ }^{\mathrm{H}}$
}

\begin{abstract}
Neuromyelitis optica spectrum disorder (NMOSD) is an uncommon antibody-mediated disease of the central nervous system. Long segments of spinal cord inflammation (myelitis), severe optic neuritis, and/or bouts of intractable vomiting and hiccoughs (area postrema syndrome) are classic presentations of the disease and may alert the clinician to the diagnosis. Untreated, approximately $50 \%$ of NMOSD patients will be wheelchair users and blind, and a third will have died within 5 years of their first attack. Unlike multiple sclerosis, a progressive clinical course is very unusual and the accrual of disability is related to relapses. Approximately $75 \%$ of patients have antibodies against aquaporin-4, a water channel expressed on astrocytes. Relapses are treated aggressively to prevent residual disability with highdose steroids and often plasma exchange. Relapse prevention is crucial and achieved with long-term immunosuppression. In this article we review the pathogenesis, clinical features, diagnosis and management of NMOSD.
\end{abstract}

KEYWORDS: Neuromyelitis optica, aquaporin-4, antibody

\section{Case vignette}

A 24-year-old male developed nausea, vomiting, and pain between his shoulder blades with leg weakness. Over the next 72 hours he developed rapidly progressive leg weakness, numbness, and urinary retention, and attended his local emergency department. He was admitted but quickly progressed to flaccid tetraplegia. There were no preceding infections or inciting events. His medical history was unremarkable except for 'cyclical vomiting syndrome'. Numerous investigations and treatments including a cholecystectomy had been unsuccessful.

He had urgent magnetic resonance imaging (MRI) of the spine, which demonstrated a long segment of high $\mathrm{T} 2$ signal and

Authors: ${ }^{A}$ post-CCT neuroimmunology fellow, Walton Centre NHS Foundation Trust, Liverpool, UK; ${ }^{B}$ neuroimmunology clinical fellow, Walton Centre NHS Foundation Trust, Liverpool, UK; ' Consultant neuroradiologist, Walton Centre NHS Foundation Trust, Liverpool, UK; DTechnical Specialist Analytical Development, Ipsen, Wrexham, UK; Econsultant neuro-ophthalmologist, Walton Centre NHS Foundation Trust, Liverpool, UK; F consultant neurologist, Walton Centre NHS Foundation Trust, Liverpool, UK; ${ }^{G}$ NMOSD clinical lead, Walton Centre NHS Foundation Trust, Liverpool, UK; ${ }^{\mathrm{H}}$ consultant paediatric neurologist and neuromyelitis optica spectrum disorder paediatric clinical lead, Alder Hey Children's NHS Foundation Trust, Liverpool, UK oedema from C1-T4 with contrast enhancement. His cerebrospinal fluid demonstrated a lymphocytic pleocytosis (white cell count 40 cells $/ \mathrm{mm}^{3}$ ) with normal protein, glucose ratio, and negative oligoclonal bands. Acute inflammatory transverse myelitis was diagnosed and he was given 1 gram of IV methylprednisolone for 5 days. His serum was sent for aquaporin-4 (AQP4) and myelin oligodendrocyte glycoprotein (MOG) antibodies (Abs). The steroid treatment did not result in significant improvement. There was concern of ascending myelitis and respiratory compromise. He was given 5 cycles of plasma exchange and continued on a tapering course of prednisolone. After 4 weeks his arm strength had improved but he remained paraplegic and was transferred to a rehabilitation facility. During his admission he complained of severe itching (to the point of excoriation) over his chest as well as painful lower limb spasms, which were successfully treated with gabapentin and carbamazepine respectively. His AQP4-Abs were positive and he was commenced on mycophenolate mofetil with prednisolone to prevent further relapses. At follow-up 3 months later he remained relapse-free but wheelchair-dependent with a spastic paraplegia. He used intermittent self-catheterisation four times per day and required regular bowel irrigation for constipation. He was commenced on baclofen for spasticity and sildenafil for erectile dysfunction. Interestingly, following plasma exchange his nausea and vomiting resolved completely suggesting that the vomiting may have been AQP4-Ab mediated area postrema syndrome.

\section{Introduction}

Neuromyelitis optica spectrum disorder (NMOSD) is an uncommon antibody-mediated disease of the central nervous system. In the UK patients often present to and are first assessed in emergency departments or acute medical units (AMU). Early diagnosis and treatment are important to reduce the risk of long-term disability and death.

The term neuromyelitis optica (derived from neuro-myélite optique aiguë) was first described by Eugène Devic and his doctoral student Fernand Gault in 1894.1 The disease was therefore previously referred to as Devic's disease. Until recently it was unclear whether neuromyelitis optica was a separate disease or merely a more severe form of 'optico-spinal' multiple sclerosis (MS). It was not until 2004 when the putative antigenic target, the aquaporin- 4 water channel was identified, and the two diseases could be reliably distinguished through the detection of AQP4-Abs. ${ }^{2}$ The latest iteration of diagnostic guidelines unify antibody-negative and positive forms under the umbrella of NMOSDs. ${ }^{3}$ 
Since the discovery of AQP4-Abs there have been over 3,000 publications on NMOSD in the PubMed database alone. More recently another antigenic target, myelin oligodendrocyte glycoprotein (MOG) has been identified in patients with NMOSD. ${ }^{4}$ This review aims to provide a basic overview of the pathogenesis, diagnosis, and management of NMOSD.

\section{Pathogenesis}

AQP4 is the most widely expressed water channel in the brain, spinal cord, and optic nerves. Within the brain, AQP4 is located in regions in contact with cerebrospinal fluid, and is specifically localised to the foot processes of astrocytes at the blood brain barrier. ${ }^{2,5} \mathrm{AQP} 4$ is also present in the collecting ducts of the kidney, parietal cells of the stomach, airways, secretory glands, and skeletal muscle. ${ }^{6}$ However, these organs are relatively protected from antibody-mediated damage due to local complement inhibitors which are absent in the brain.?

AQP4-Abs are predominantly of the IgG1-isotype. Experimental data suggest that AQP4-Abs induce interleukin-6 (IL-6) production in astrocytes expressing AQP4, and that IL-6 signalling to endothelial cells reduces blood-brain barrier function. ${ }^{8}$ Once bound to the extracellular domain of the AQP4 receptor, AQP4-Abs result in complement- and cell-mediated astrocytic damage, in addition to internalisation of the glutamate transporter EAAT-2. ${ }^{9}$ The astrocyte is subsequently rendered powerless, ultimately culminating in withdrawal of support for surrounding cells such as oligodendrocytes and neurons. Granulocyte infiltration ensues, matched by oligodendrocyte damage and demyelination (Fig 1). ${ }^{10}$ In contrast to MS, the demyelination that is seen in NMOSD is a secondary event and occurs as a consequence of primary damage to astrocytes.

\section{Epidemiological and genetic factors}

The reported incidence and prevalence of NMOSD are dependent on geographical location and ethnicity. Asians and those of African ancestry are at increased risk, with high mortality rates reported in the latter. ${ }^{11,12}$ The incidence and prevalence of NMOSD ranges from $0.05-0.40$ and $0.52-4.4$ per 100,000 people respectively. ${ }^{13}$ In contrast to MS, a latitude gradient for the prevalence of NMOSD has not been substantiated. ${ }^{14}$ As with many autoimmune diseases, females are more susceptible than males (3:1-9:1). ${ }^{15}$ A female hormonal basis for this association may be a factor but requires further study. ${ }^{16}$ The median age at presentation is 39 years, but $15-20 \%$ of patients may present to paediatricians (under 16 years) or elderly care physicians (greater than 65 years). ${ }^{15}$ Clustering of NMOSD in families is rare but recognised, suggesting complex genetic susceptibility. ${ }^{17}$ A recent whole-genome sequence study identified genetic variants in the major histocompatibility region that contribute to NMOSD aetiology. ${ }^{18}$ Approximately one in four patients with AQP4-Ab positive NMOSD have another coexisting autoimmune disease, eg myasthenia gravis, systemic lupus erythematosus (SLE), Sjogren's and coeliac disease. ${ }^{19-22}$ Accordingly, specialties such as rheumatology should have a low threshold for testing AQP4-Abs; eg SLE patients with intractable vomiting/hiccoughs or optic neuritis without obvious cause. In one series $46 \%$ of myelitis cases thought secondary to antiphospholipid/SLE syndrome were subsequently found to harbour AQP4-Abs. ${ }^{23}$
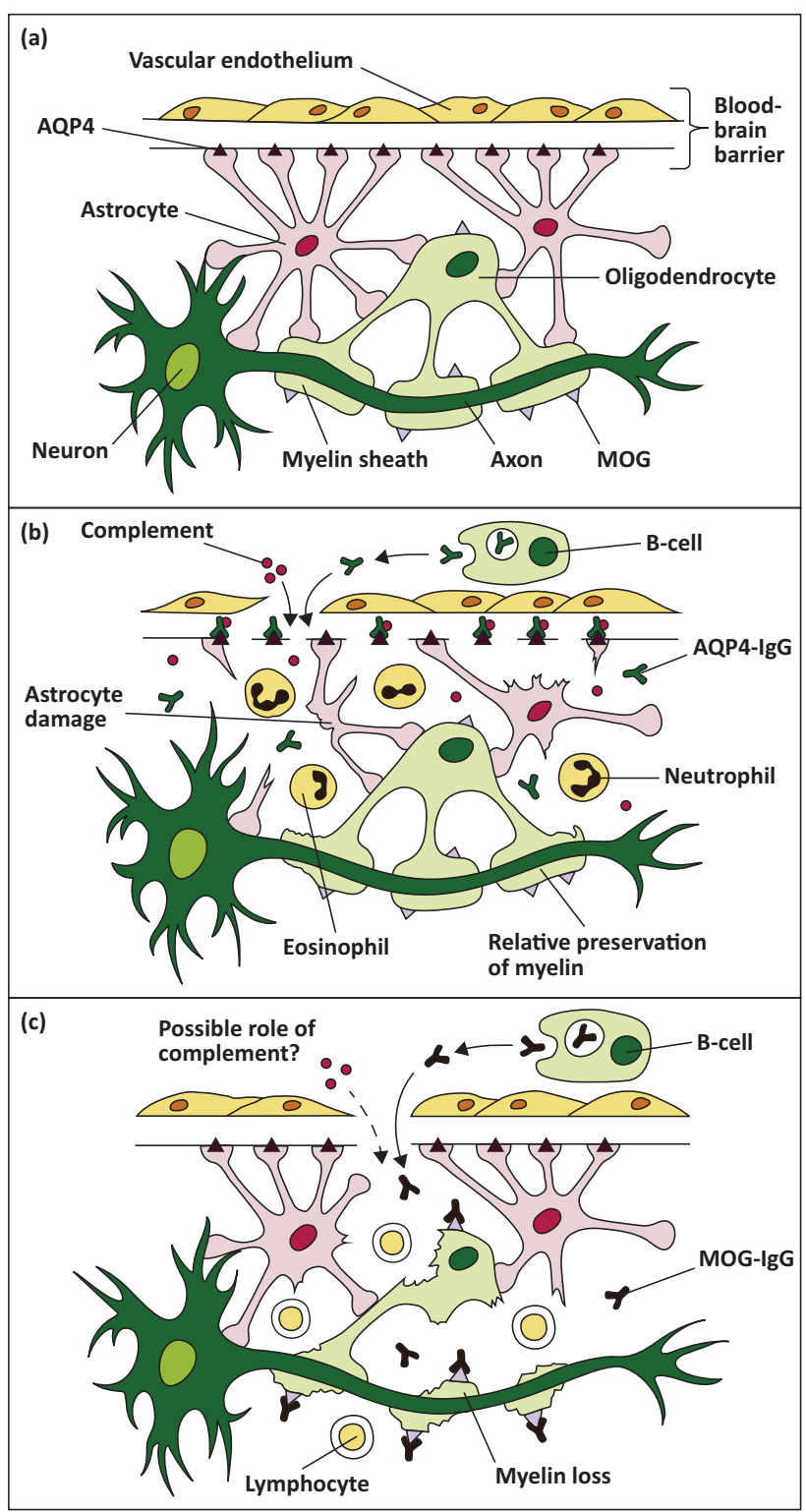

Fig 1. (a) This figure illustrates the sites of expression of aquaporin-4 (AQP4) and myelin oligodendrocyte glycoprotein (MOG) in the central nervous system (CNS). AQP4 is expressed on astrocyte 'foot-like' processes at the blood-brain barrier. MOG is expressed on oligodendrocytes on the outermost lamellae of myelin sheaths. (b) AQP4-Abs (IgG) are produced systemically by mature B-cells, and upon crossing the blood-brain barrier, activate complement-mediated astrocyte damage. There is relative preservation of myelin initially. The inflammatory milieu consists of neutrophils and eosiniphils. (c) MOG-Abs (IgG) are also produced outside the CNS, and causes demyelination by mechanisms that are poorly understood. Reprinted with permission from Whittam D, Wilson M, Hamid S et al. What's new in neuromyelitis optica? A short review for the clinical neurologist. J Neurol 2017;264:2330-44.

\section{Clinical presentation}

Table 1 summarises the 2015 diagnostic criteria for NMOSD, which incorporates classic clinical presentations of the disease. ${ }^{3}$ These involve regions of the central nervous system (CNS) where 
Table 1. Summary of 2015 international diagnostic criteria for neuromyelitis optica spectrum disorder ${ }^{3}$

\section{Diagnosis with AQP4-Abs}

at least one core clinical characteristic

positive AQP4-Abs with best available method

exclusion of alternative diagnosis (eg sarcoidosis, neoplastic/ paraneoplastic, vascular, chronic infection).

\section{Diagnosis without AQP4-Abs/unknown status}

at least two core clinical characteristics resulting from one or more clinical attacks and fulfilling the following:

at least one of optic neuritis, LETM, APS

dissemination in space (two or more different core clinical characteristics)

> fulfilment of additional MRI requirements as applicable

negative for AQP4-Abs with best available method, or testing unavailable

exclusion of alternative diagnoses.

\section{Core clinical characteristics}

optic neuritis

acute myelitis

APS

brainstem syndrome

symptomatic narcolepsy or acute diencephalic syndrome with NMOSD-typical diencephalic MRI lesions

symptomatic cerebral syndrome with NMOSD-typical brain lesions.

\section{Additional MRI requirements for NMOSD without AQP4 Abs/unknown status}

acute optic neuritis: normal or only non-specific white matter lesions on MRI brain; or optic nerve MRI with T2-hyperintense lesion or T1-weighted gadolinium-enhancing lesion extending over $>1 / 2$ optic nerve length or optic chiasm involvement

acute myelitis: MRI spinal cord demonstrating attackassociated lesion spanning $\geq 3$ contiguous vertebral segments (LETM); or $\geq 3$ contiguous segments of focal cord atrophy with previous history of acute myelitis

$>$ APS: dorsal medulla/area postrema lesion on MRI brain

$>$ acute brainstem syndrome: periependymal brainstem lesions.

$\mathrm{APS}=$ area postrema syndrome; $\mathrm{AQP4}-\mathrm{Abs}=$ aquaporin- 4 antibodies; $\mathrm{LETM}=$ longitudinally extensive transverse myelitis; NMOSD = neuromyelitis optica spectrum disorder; $\mathrm{MRI}=$ magnetic resonance imaging.

AQP4 is most abundantly expressed; spinal cord (longitudinally extensive transverse myelitis), optic nerve (optic neuritis), dorsal medulla (area postrema syndrome), brainstem (acute brainstem syndromes), and thalamus/hypothalamus (acute diencephalic syndromes eg symptomatic narcolepsy). Attacks are frequently severe and often reach nadir in less than a week. Longitudinally extensive transverse myelitis (LETM) is the most specific presentation of NMOSD and is uncommon in MS (Fig 2a). LETM typically consists of inflammation affecting the central

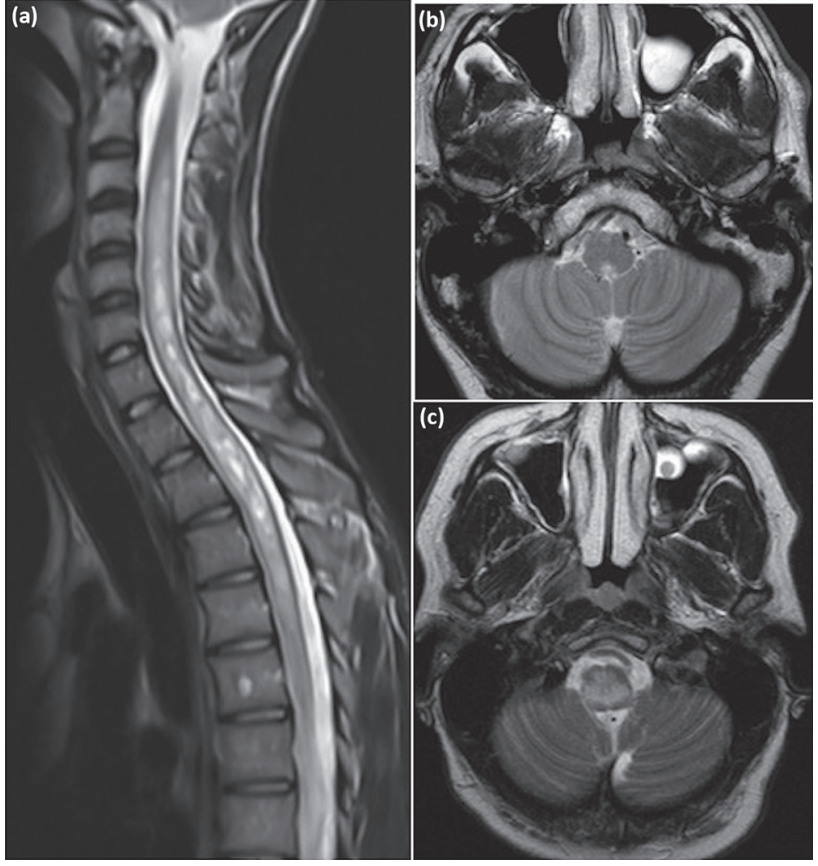

Fig 2. Magnetic resonance imaging (MRI) in neuromyelitis optica. (a) Longitudinally extensive transverse myelitis - sagittal T2 weighted image of cervicothoracic spine demonstrating $T 2$ hyperintensity extending longitudinally from C2-T4. (b) Area postrema syndrome - axial T2 weighted image demonstrating dorsal T2 signal hyperintensity in the medulla. (c) Brainstem syndrome - axial T2 weighted image demonstrating diffuse symmetrical T2 signal hyperintensity in the medulla.

gray matter, extending over three or more contiguous vertebral bodies. ${ }^{24}$ LETM often results in para- or tetraplegia depending on the spinal cord level involved. A sensory level and bladder involvement are useful distinguishing features from other causes of rapid evolving weakness such as Guillain-Barré syndrome. Table 2 summarises the differential diagnosis of transverse myelitis. Other useful clinical clues include intense itching (due to inflammation of itch specific fibres in the spinothalamic tract) and tonic spasms (brief recurrent, usually painful episodes of increased muscle tone with abnormal posturing of the affecting limb). ${ }^{25,26}$ Importantly, up to $14 \%$ of NMOSD patients may also present with short spinal cord lesions, which may mimic MS. ${ }^{27}$ In such cases the absence of MS-typical brain lesions eg 'Dawson's fingers' should increase the suspicion of NMOSD.

Optic neuritis (ON) in NMOSD is also typically longitudinally extensive and has a predilection for posterior optic nerve segments, particularly the optic chiasm. Bilateral simultaneous involvement and painful severe visual loss with poor recovery $(<6 / 60)$ are often clues to the diagnosis.

Area postrema syndrome (APS) results in intractable nausea, vomiting and/or hiccoughs secondary to inflammation in the emetic reflex centre located in the rhomboid fossa of the 4 th ventricle (Fig 2b). ${ }^{28}$ Patients may initially present with suspected gastroenteritis or cyclical vomiting syndrome. APS is the initial presenting feature of NMOSD in approximately $12 \%$ of cases. ${ }^{11}$

Acute brainstem syndromes overlap with APS but also include patients who present with oculomotor dysfunction (eg diplopia 
Table 2. Differential diagnosis of transverse myelitis

Structural Degenerative disc disease, tumours (primary or secondary), syrinx, abscess

Autoimmune/ MS, GBS, AQP4-Ab, MOG-Ab, GFAP-Ab, inflammatory sarcoid, stiff-person syndrome, SLE, Behcet's, Sjogren's, MCTD

Vascular Anterior spinal artery occlusion, dural arteriovenous fistula, CNS vasculitis

Nutritional B12/folate/copper (zinc intoxication), vitamin E deficiency

Viral/post-viral DNA viruses eg CMV, varicella-zoster, HSV-2, EBV

RNA viruses eg HIV, HTLV-1/2, polio, influenza, measles, mumps, dengue, West Nile, enterovirus-D68, 70/71, hepatitis A/C

Other infections Mycoplasma, Borrelia burgdorferi, Treponema pallidum, aspergillus, neurocysticercosis, schistosoma, angiostrongylosis

Genetic/inborn eg Leukodystrophies, peroxisomal disorders, metabolic errors biotinidase deficiency, hereditary spastic paraplegias, dopa-responsive dystonia, Friedreich's ataxia, Hexosaminidase deficiency

Paraneoplastic Amphiphysin-, Ri-, CV2/CRMP5-, Ma1/2-, syndromes Hu-antibodies

Toxic

Post-radiation

Nitrous oxide

Heroin

Electrical injury

Decompression sickness

Anterior horn Amyotrophic lateral sclerosis

cell disease

Primary lateral sclerosis

Poliomyelitis

$\mathrm{Ab}=$ antibody; $\mathrm{AQP} 4=$ aquaporin-4; $\mathrm{CMV}=$ cytomegalovirus; $\mathrm{CNS}$ = central nervous system; CV2/CRMP5 = collapsin response mediator protein 5; DNA = deoxyribonucleic acid; EBV = Epstein-Barr virus; GBS = Guillain-Barre syndrome; GFAP = glial fibrillary acid protein; HSV = herpes simplex virus; $\mathrm{HTLV}=$ human T-lymphocytic virus; $\mathrm{MCTD}=$ mixed connective tissue disease; $\mathrm{MOG}=$ myelin oligodendrocyte glycoprotein; MS = multiple sclerosis; SLE = systemic lupus erythematosus; RNA = ribonucleic acid.

and nystagmus), or other cranial nerve palsies, depending on location (Fig 2c). ${ }^{29}$

AQP4 is highly expressed in the hypothalamic periventricular regions and bilateral lesions may affect hypothalamic hypocretin neuronal function. Several cases of narcolepsy, with diencephalic lesions and low cerebrospinal fluid (CSF) hypocretin levels have been reported. ${ }^{30}$

Although the term neuromyelitis optica might suggest exclusive spinal cord and optic nerve inflammation, brain involvement can be seen in $60 \%$ of patients, though the majority of observed changes are non-specific. ${ }^{31}$ Cerebral involvement may be asymptomatic, but can cause encephalopathy, seizures, and hemiparesis. Lesions often enhance with contrast on MRI and maybe mistaken for a primary CNS tumour. AQP4 is expressed at high density in the periependymal regions outlining the ventricular system, and characteristic lesions involving this region may be seen.

\section{Diagnostic considerations}

The presence of one of the six core clinical characteristics (LETM, ON, APS, symptomatic brainstem, diencephalic, or cerebral syndromes) with AQP4-Abs is sufficient to make a diagnosis of NMOSD. In seronegative cases the criteria are more stringent and MRI requirements must also be fulfilled. For instance, at least three vertebral segments of spinal cord inflammation/atrophy must be demonstrated in LETM and in APS, a dorsal medulla lesion must be present. ${ }^{3}$ Lesions considered typical for MS can be seen in approximately $10 \%$ of cases but there are specific MRI features (lesions adjacent to body of lateral ventricle and in inferior temporal lobe, S-shaped U-fibre lesions, and presence of Dawson's finger-type lesions) that help distinguish MS from NMOSD. ${ }^{31,32}$ Table 3 summarises the differences between NMOSD and MS. Of available AQP4-Ab assays, the cell based assay (CBA) has the highest sensitivity $(76.7 \%)$ and specificity $(99.8 \%) .{ }^{33}$ The impact and discriminatory power of detecting AQP4-Abs has likely contributed to the increase in rates of NMOSD diagnosis. ${ }^{34,35}$ Furthermore, following an episode of LETM the presence of AQP4-Abs confers a $50 \%$ risk of further relapse in the next 12 months. ${ }^{36}$ The presence of non-organ specific antibodies eg antinuclear, anti-double-stranded DNA, anti-Ro, anti-La may also be seen. ${ }^{3,22}$ Cerebrospinal fluid typically contains a mixed pleocytosis (lymphocytes, neutrophils, and monocytes) with a median cell count of 19 cells/ $\mu$ (range: 6-380). Unlike MS, CSF-restricted oligoclonal bands are uncommon. ${ }^{37}$ Other useful investigations include visual evoked potentials and optical coherence tomography. ${ }^{38}$

\section{MOG-antibodies}

In 2012 some patients with seronegative NMOSD were found to have MOG-Abs. MOG is expressed on the surface of oligodendrocytes and myelin in the CNS. Though postulated as a candidate autoantigen in CNS autoimmunity for almost 30 years, the advent of the CBA has facilitated the detection of Abs against human MOG expressed in its conformational state, which is essential for diagnostic relevance. MOG-Abs account for approximately $40 \%$ of NMOSD patients who are seronegative for AQP4. ${ }^{39}$ It is therefore reasonable when suspecting NMOSD to test for both AQP4-Abs and MOG-Abs (Table 2).

The most frequent presentation of MOG-Ab-associated disease is acute disseminated encephalomyelitis (ADEM) in children under 7 years, and optic neuritis in older children and adults. ${ }^{40,41}$ MOG-Abs appear to have a predilection for inflammation of the anterior segments of the optic nerve, in contrast to NMOSD with AQP4-Abs, which tends to involve the chiasm and optic tracts. ${ }^{42}$ Visual outcomes are generally better but visual disability is not uncommon. Although motor outcomes after myelitis are also better, patients can be left with significant sphincter and erectile dysfunction. ${ }^{41}$ Seizures can also occur as part of an encephalitislike presentation with cortical involvement, more commonly than with AQP4-Abs. ${ }^{43,44}$ Importantly a larger proportion of patients with MOG-Abs may have monophasic disease with transient presence of MOG-Abs, meaning that long-term immunosuppression is not always required..$^{41}$ It is becoming increasingly clear that despite the clinical overlap between CNS 
Table 3. Differences between neuromyelitis optica spectrum disorder and multiple sclerosis

Frequency of disease
Latitude gradient
Female sex, \%
Ethnic variation
Age at onset
Progressive course
Coexistent autoimmune disease
Tissue involvement
Necrosis/cavitation
Leukocyte infiltrate
Perivascular IgG and complement
Attack severity
Spinal cord

\section{MS}

Common

Present

70

Common in Caucasians

20-40

Common

Rare

White matter

Rare

T and B lymphocytes

Common

Often mild

Short-segment peripheral cord lesions

May be asymptomatic

Optic nerve

Brainstem

Diencephalon

Corpus callosum

Cerebral hemispheres

CSF

Permanent disability

Treatment

Relapse

Long-term
Short segment inflammation, anterior

Unilateral

Good recovery

Any location

Ventral or dorsal pontine lesion

Clearly defined borders

Uncommon

Very common

Small lesions

Anterior/posterior CC

Ovoid lesions perpendicular to lateral ventricle (Dawson's fingers)

Lesion adjacent to body of lateral ventricle and in inferior temporal lobe

Juxtacortical U-fibre lesions

Mild pleocytosis; mononuclear

OCBs $85 \%$

Usually in later progressive disease phase

Consider steroids ( 5 days) for severe acute relapses

In the UK disease modifying therapies are considered in active MS ( $\geq 2$ relapses in 2 years)

\section{NMOSD}

Rare

Not proven

90

Common in Africans and Asians

40-60

Rare

Common: MG, SLE, Sjogren's, thyroid, APL

White and gray matter

Common

Neutrophils and eosinophils

Common

Often severe

Usually LETM, central cord involvement

Extension into medulla

Usually symptomatic

Acute T1 hypointensity

Long segment inflammation posterior

Unilateral/bilateral

Poor recovery

Area postrema/dorsal medulla MRI lesion

May be contiguous with spinal lesion

Hypothalamic, thalamic, periependymal 3rd ventricle region

Uncommon

Long lesions

Callosal-septal interface in middle and posterior thirds of CC

Large, confluent subcortical or deep white matter lesions

Long corticospinal tract lesions

Occasional prominent pleocytosis, lymphocytes, PMN and mononuclear cells OCBs uncommon

Usually attack-related

High dose steroids

Earlier treatment is essential. Escalation to PLEX if needed

AZA/MMF/RTX for all AQP4-Ab positive NMOSD or relapsing seronegative NMOSD

$\mathrm{APL}=$ antiphospholipid syndrome; $\mathrm{AQP4}-\mathrm{Ab}=$ aquaporin-4 antibodies; $\mathrm{AZA}=$ azathioprine; $\mathrm{CC}=$ corpus callosum; $\mathrm{CSF}=$ cerebrospinal fluid; Ig = immunoglobulin; LETM = longitudinally extensive transverse myelitis; $M A C=$ membrane attack complex; $M G=$ myasthenia gravis; $M M F=$ mycophenolate mofetil; $M R I=$ magnetic resonance imaging; $\mathrm{OCBs}=$ oligoclonal bands; $\mathrm{PLEX}=$ plasma exchange; $\mathrm{PMN}=$ polymorphonuclear cells; RRMS = relapsing remitting multiple sclerosis; RTX = rituximab; SLE = systemic lupus erythematosus. 


Table 4. Which patients should be tested for
aquaporin-4 antibodies?
When to test for aquaporin-4 antibodies ${ }^{\dagger}$
1. LETM or $\geq 3$ contiguous segments of focal cord atrophy on
MRI spine
2. 'Idiopathic' acute transverse myelitis without MS features
3. Severe unilateral optic neuritis with poor recovery, and/or
involving posterior visual pathway, eg optic chiasm
4. Bilateral simultaneous or sequential optic neuritis
5. Intractable nausea, vomiting, hiccoughs without clear
explanation
6. Dorsal medullary lesion on MRI brain
7. Diencephalic clinical syndrome
8. Cryptogenic leukoencephalopathy
9. MS apparently unresponsive/worsening after starting MS
disease modifying therapies
10. CNS inflammation atypical for MS without CSF oligoclonal
bands
If initial testing is negative, the sensitivity of AQP4-Ab detection
is increased with repeat testing 3-6 months later
It is reasonable to test for myelin oligodendrocyte glycoprotein antibodies
nervous system; CSF = cerebrospinal fluid; LETM = longitudinally extensive
sclerosis. Adapted from Whittam et al ${ }^{60}$

inflammation with AQP4- and MOG-Abs there are important biological differences including; disease mechanism, clinical phenotype, relapse rates, and treatment response. It has therefore been suggested that the 2015 NMOSD diagnostic criteria may need revision to account for these important differences. ${ }^{45}$

\section{Acute treatment}

Acute treatment of an NMOSD attack consists of high dose steroids (HDS), typically 1 gram of intravenous methylprednisolone daily for 5 days; oral prednisolone $1 \mathrm{mg} / \mathrm{kg}$ is then continued for weeks, followed by gradual taper over months. Earlier treatment is ideal and with severe neurological deficits, if improvement is not seen within days of HDS, plasma exchange (PLEX; 5 cycles) should be commenced. Escalation therapy has been shown to increase response/remission rates and should be offered in appropriate cases. ${ }^{46}$ There is some data to support PLEX as firstline treatment for relapses, particularly myelitis, but prospective randomised trials are required to substantiate this observation. ${ }^{47}$ Thromboprophylaxis is advised, particularly in non-ambulant patients with myelitis.

\section{Long-term treatment}

Untreated, approximately $50 \%$ of NMOSD patients will be wheelchair users and blind, and a third will have died within 5 years of their first attack. ${ }^{48}$ We therefore treat all patients with AQP4-Abs at their first attack with long-term immunosuppression. The most commonly used first line immunosuppressants (IS) in
NMOSD are mycophenolate mofetil (MMF; 2-3 grams/day) and azathioprine (AZA; $2.5-3 \mathrm{mg} / \mathrm{kg}$ ). Retrospective data suggest that MMF may be superior to AZA (reduction in relapse rate $87.4 \%$ versus $72.1 \%$ respectively) but prospective data are lacking. ${ }^{49} \mathrm{AZA}$ is often selected in younger female patients as $M M F$ is contraindicated in pregnancy. It should also be noted that MMF can have spermatotoxic effects. Oral prednisolone $(5-10 \mathrm{mg})$ is often given long-term as the combination may be more protective than MMF/AZA alone. ${ }^{50}$ Importantly, steroids should be given to cover the period of time it takes for AZA/MMF to take full effect (with AZA a $5 \mathrm{fL}$ rise in mean cell volume or mild absolute lymphocyte count suppression are useful indicators of this). Currently in the UK, the B-cell depleting monoclonal antibody, rituximab (RTX) is used as a second-line medication. $\mathrm{RTX}$ reduces relapse rates up to $88.2 \%$ and is either given 6 -monthly or according to monitored B-cell counts (CD19 ${ }^{+}$lymphocytes). ${ }^{51}$ Our practice is to re-treat if CD19 counts rise above $1 \%$ of the total B-cell population, but other treatment paradigms exist. ${ }^{50}$ We also periodically check serum immunoglobulin levels as some patients may develop symptomatic secondary hypogammaglobulinaemia. ${ }^{52}$

Other IS that are occasionally used include tocilizumab, methotrexate, cyclophosphamide, mitoxantrone, intravenous immunoglobulins, tacrolimus, and ciclosporin. Importantly, many of the disease modifying drugs used in the treatment of MS have been associated with futility or exacerbation in NMOSD, and should be avoided eg beta-interferon, fingolimod, and natalizumab. $^{53-55}$

A detailed review of general symptom management in NMOSD is beyond the scope of this article but three symptoms deserve special mention. Tonic spasms following transverse myelitis can often effectively be treated with a low dose of carbamazepine. ${ }^{50}$ Neuropathic pain is more severe and disabling as compared with MS and early involvement of a local pain team is helpful. ${ }^{56}$ Moderate to severe depression and fatigue are common, associated with neuropathic pain and challenging to treat. ${ }^{57}$ Ideally a multidisciplinary approach with regular review of the effectiveness of pharmacotherapies is recommended.

Preliminary results of the first multi-centre, randomised, double-blind, placebo-controlled trial in NMOSD were recently reported (NCT01892345). ${ }^{58}$ Publication of the full trial results are awaited but the initial results support the use of eculizumab in the armamentarium of drugs for NMOSD, though its expense is a concern. At the time of review, clinical trials assessing the efficacy of SA237 (satralizumab - anti-interleukin- 6 receptor; NCT02073279 and NCT0202884) and MEDI-551 (inebilizumab humanised monoclonal antibody against CD19; NCT002200770) also reported positive outcomes with reduction in relapse rates between $70-90 \%$.

\section{UK NMOSD service}

The UK NMOSD service (www.nmouk.nhs.uk) is funded by NHS England Highly Specialised Services, and aims to provide highquality, up-to-date, evidence-based multidisciplinary care. The team consists of adult and paediatric neurologists, clinical fellows, nurse specialists, occupational therapists, physiotherapists, ophthalmologists/orthoptists, dieticians, and clinical psychologists. The service operates out of the Walton Centre Foundation Trust in Liverpool and John Radcliffe Hospital in Oxford but telephone/ 
email advice, home visits, and satellite clinics help to ensure countrywide accessibility of care. Referrals are accepted from GPs and all medical specialities. The NMOSD service has also produced guidelines to unify treatment approaches across the UK, runs educations days, webinars, and provides direct patient access. Relapse rates and morbidity from NMOSD have both improved since the introduction of the service in $20100^{59}$ The service has an active research portfolio and has facilitated the development of the NMO Spectrum-UK charity (www.nmouk.org). It has also received a Royal College of Physicians Excellence in Patient Care Award in 2016.

\section{Take-home messages}

> Neuromyelitis optica spectrum disorder (NMOSD) is a relapsing central nervous system disease associated with aquaporin-4 antibodies.

> Common presentations include longitudinally extensive myelitis, severe optic neuritis, and area postrema syndrome.

$>$ Prompt and aggressive treatment of relapses with high dose steroids +/- plasma exchange improves outcomes.

$>$ All patients with aquaporin- 4 antibodies should be immunosuppressed indefinitely to prevent further attacks.

> The NMOSD service based in Liverpool (Walton Centre NHS Foundation Trust) and Oxford (John Radcliffe Hospital) provides a quaternary service for all NMOSD patients in the UK. Referrals are accepted from GPs and all medical specialties.

\section{Conflicts of interest}

Dr Jacob has received research grants from Biogen Idec, Alexion Pharmaceuticals and speaker fees from Biogen, Chugai, SanofiGenzyme, and Terumo-BCT.

\section{References}

1 Jarius S, Wildemann B. The history of neuromyelitis optica. J Neuroinflammation 2013;10:8.

2 Lennon VA, Wingerchuk DM, Kryzer T] et al. A serum autoantibody marker of neuromyelitis optica: distinction from multiple sclerosis. Lancet 2004;364:2106-12.

3 Wingerchuk DM, Banwell B, Bennett JL et al. International consensus diagnostic criteria for neuromyelitis optica spectrum disorders. Neurology 2015; 85:177-89.

4 Kitley ], Woodhall M, Waters P et al. Myelin-oligodendrocyte glycoprotein antibodies in adults with a neuromyelitis optica phenotype. Neurology 2012;79:1273-7.

5 Lennon VA, Kryzer T], Pittock S], Verkman AS, Hinson SR. IgG marker of optic-spinal multiple sclerosis binds to the aquaporin-4 water channel. J Exp Med 2005;202:473-7.

6 Verkman AS. Aquaporins in clinical medicine. Ann Rev Med 2012:63:303-16.

7 Saadoun S, Papadopoulos MC. Role of membrane complement regulators in neuromyelitis optica. Mult Scler 2015;21:1644-54.

8 Takeshita Y, Obermeier B, Cotleur AC, et al. Effects of neuromyelitis optica-IgG at the blood-brain barrier in vitro. Neurol Neuroimmunol Neuroinflamm 2017:4:e311.

9 Hinson SR, Pittock SJ, Lucchinetti CF et al. Pathogenic potential of IgG binding to water channel extracellular domain in neuromyelitis optica. Neurology 2007:69:2221-31.

10 Hinson SR, Roemer SF, Lucchinetti CF et al. Aquaporin-4-binding autoantibodies in patients with neuromyelitis optica impair glutamate transport by down-regulating EAAT2. J Exp Med 2008;205:2473-81.
11 Pittock S], Lucchinetti CF. Neuromyelitis optica and the evolving spectrum of autoimmune aquaporin-4 channelopathies: a decade later. Annals of the New York Academy of Sciences 2016;1366:2039.

12 Mealy MA, Kessler RA, Rimler Z, et al. Mortality in neuromyelitis optica is strongly associated with African ancestry. Neurol Neuroimmunol Neuroinflamm 2018;5:e468.

13 Pandit L, Asgari N, Apiwattanakul M et al. Demographic and clinical features of neuromyelitis optica. Mult Scler ] 2015;21:845-53.

14 Masahiro M, Satoshi K, Fridemann P. Worldwide prevalence of neuromyelitis optica spectrum disorders. J Neurol Neurosurg Psychiatry 2018:89:555-6.

15 Quek AML, Mckeon A, Lennon VA et al. Effects of age and sex on aquaporin-4 autoimmunity. Arch Neurol 2012;69:1039-43.

16 Bove R, Elsone L, Alvarez E et al. Female hormonal exposures and neuromyelitis optica symptom onset in a multicentre study. Neurol Neuroimmunol Neuroinflamm 2017;4:e339.

17 Matiello M, Kim HJ, Kim W et al. Familial neuromyelitis optica. Neurology 2010;75:310-5.

18 Estrada K, Whelan CW, Zhao F et al. A whole-genome sequence study identifies genetic risk factors for neuromyelitis optica. Nature Communications 2018;9:1929.

19 Pittock SJ, Lennon VA, de Seze J et al. Neuromyelitis optica and non-organ specific autoimmunity. Arch Neurol 2008;65:78-83.

20 Jacob S, Zarei M, Kenton A et al. Gluten sensitivity and neuromyelitis optica: two case reports. J Neurol Neurosurg Psychiatry 2005;76:1028-30

21 Leite MI, Coutinho E, Lana-Peixoto M et al. Myasthenia gravis and neuromyelitis optica spectrum disorder: A multicenter study of 16 patients. Neurology 2012;78:1601-7.

22 Jarius S, Ruprecht K, Wildemann B et al. Contrasting disease patterns in seropositive and seronegative neuromyelitis optica: A multicentre study of 175 patients. J Neuroinflammation 2012;9:14.

23 Guerra H, Pittock S], Moder KG et al. Frequency of aquaporin-4 immunoglobulin $\mathrm{G}$ in longitudinally extensive transverse myelitis with antiphospholipid antibodies. Mayo Clin Proc 2018;10:1-6.

24 Wingerchuk DM, Lennon VA, Pittock S] et al. Revised diagnostic criteria for neuromyelitis optica. Neurology 2006;66:1485-9.

25 Elsone L, Townsend T, Mutch K et al. Neuropathic pruritus (itch) in neuromyelitis optica. Mult Scler ] 2012;19:475-9.

26 Usmani N, Bedi G, Lam BL et al. Association Between Paroxysmal Tonic Spasms and Neuromyelitis Optica. Arch Neurol 2012;69:121-4.

27 Flanagan EP, Weinshenker BG, Krecke KN et al. Short myelitis lesions in aquaporin-4-IgG-positive neuromyelitis optica spectrum disorders. JAMA Neurol 2015;72:81-7.

28 Duvernoy HM, Risold PY. The circumventricular organs: An atlas of comparative anatomy and vascularization. Brain Research Reviews 2007:56:119-47.

29 Kremer L, Mealy M, Jacob A et al. Brainstem manifestations in neuromyelitis optica: a multicenter study of 258 patients. Mult Scler ] 2013:20:843-7.

30 Kanbayashi T, Shimohata T, Nakashima I et al. Symptomatic narcolepsy in patients with neuromyelitis optica and multiple sclerosis: new neurochemical and immunological implications. Arch Neurol 2009;66:1563-6.

$31 \mathrm{Kim} \mathrm{H}$, Friedermann P, Lana-Peixoto MA et al. MRI characteristics of neuromyelitis optica spectrum disorder. Neurology 2015;84:1165-73

32 Jurýnczyk M, Tackley G, Kong Y et al. Brain lesion distribution criteria distinguish MS from AQP4-antibody NMOSD and MOGantibody disease. J Neurol Neurosurg Psychiatry 2016;88:132-6.

33 Waters PJ, Pittock SJ, Bennett JL et al. Evaluation of aquaporin-4 antibody assays. Clin Exp Neuroimmunol 2014;5:290-303.

34 Hamid SH, Elsone L, Mutch K et al. The impact of 2015 neuromyelitis optica spectrum disorders criteria on diagnostic rates. Mult Scler 2017;23:228-33. 
35 Hyun J-W, Jeong IH, Joung A et al. Evaluation of the 2015 diagnostic criteria for neuromyelitis optica spectrum disorder. Neurology 2016;86:1772-9.

36 Weinshenker BG, Wingerchuk DM, Vukusic $S$ et al. Neuromyelitis optica IgG predicts relapse after longitudinally extensive transverse myelitis. Ann Neurol 2006;59:566-9.

37 Jarius S, Paul F, Franciotta D et al. Cerebrospinal fluid findings in aquaporin- 4 antibody positive neuromyelitis optica: results from 211 lumbar punctures. J Neurol Sci 2011;306:82-90.

38 Bennett JL, de Seze J, Lana-Peixoto $M$ et al. Neuromyelitis optica and multiple sclerosis: Seeing differences through optical coherence tomography. Mult Scler 2015;21:678-88.

39 Hamid SHM, Whittam D, Mutch K et al. What proportion of AQP4IgG-negative NMO spectrum disorder patients are MOG-IgG positive? A cross sectional study of 132 patients. J Neurol 2017;264:2088-94.

40 Hacohen Y, Wong YY, Lechner $C$ et al. Disease course and treatment responses in children with relapsing myelin oligodendrocyte glycoprotein antibody-associated disease. JAMA Neurol 2018;75:478-87.

41 Juryńczyk M, Messina S, Woodhall MR et al. Clinical presentation and prognosis in MOG-antibody disease: a UK study. Brain 2017; 140:3128-38.

42 Ramanathan S, Prelog K, Barnes EH et al. Radiological differentiation of optic neuritis with myelin oligodendrocyte glycoprotein antibodies, aquaporin-4 antibodies, and multiple sclerosis. Mult Scler ] 2016;22:470-82.

43 Hamid S, Whittam D, Saviour M et al. Seizures and encephalitis in myelin oligodendrocyte glycoprotein IgG disease vs aquaporin-4 IgG disease. JAMA Neurol 2018;75:65-71.

44 Ogawa R, Nakashima I, Takahashi T et al. MOG antibody-positive, benign, unilateral, cerebral cortical encephalitis with epilepsy. Neurol Neuroimmunol Neuroinflamm 2017;4:e322.

45 Hacohen Y, Palace J. Time to separate MOG-Ab-associated disease from AQP4-Ab-positive neuromyelitis optica spectrum disorder. Neurology 2018;90:947-8.

46 Kleiter I, Gahlen A, Borisow N et al. Neuromyelitis optica: Evaluation of 871 attacks and 1,153 treatment courses. Ann Neurol 2016;79:206-16.

47 Weinshenker BG. What is the optimal sequence of rescue treatments for attacks of neuromyelitis optica spectrum disorder? Ann Neurol 2016;79:204-5.

48 Wingerchuk DM, Hogancamp WF, O'Brien PC et al. The clinical course of neuromyelitis optica (Devic's syndrome). Neurology 1999;53:1107-14.
49 Mealy MA, Wingerchuk DM, Palace ] et al. Comparison of relapse and treatment failure rates among patients with neuromyelitis optica: multicenter study of treatment efficacy. JAMA Neurol 2014:71:324-30.

50 Palace J, Leite I, Jacob A. A practical guide to the treatment of neuromyelitis optica. Pract Neurol 2012;12:209-14.

51 Kim S-H, Huh S-Y, Lee S] et al. A 5-year follow-up of rituximab treatment in patients with neuromyelitis optica spectrum disorder. JAMA Neurol 2013;70:1110-7.

52 Tallantyre EC, Whittam DH, Jolles S et al. Secondary antibody deficiency: a complication of anti-CD20 therapy for neuroinflammation. J Neurol 2018;265:1115-22.

$53 \mathrm{Min} \mathrm{J}-\mathrm{H}$, Kim BJ, Lee KH. Development of extensive brain lesions following fingolimod (FTY720) treatment in a patient with neuromyelitis optica spectrum disorder. Multiple Sclerosis Journal 2011;18:113-5.

54 Jacob A, Hutchinson M, Elsone L et al. Does natalizumab therapy worsen neuromyelitis optica? Neurology 2012;79:1065-6.

55 Palace J, Leite MI, Nairne et al. Interferon beta treatment in neuromyelitis optica: increase in relapses and aquaporin 4 antibody titers. Arch Neurol 2010;67:1016-7.

56 Kanamori $Y$, Nakashima I, Takai $Y$ et al. Pain in neuromyelitis optica and its effect on quality of life: a cross-sectional study. Neurology 2011;77:652-8.

57 Chavarro VS, Mealy MA, Simpson A et al. Insufficient treatment of severe depression in neuromyelitis optica spectrum disorder. Neurol Neuroimmunol Neuroinflamm 2016;3:e286.

58 Business Wire. Alexion announces successful Phase 3 PREVENT study of Soliris $®$ (Eculizumab) in patients with neuromyelitis optica spectrum disorder (NMOSD). Business Wire. www.businesswire.com/news/home/20180924005 283/en/Alexion- AnnouncesSuccessful-Phase-3-PREVENT-Study [Accessed 6 November 2018].

59 Whittam D, Hamid S, Linaker S et al. Relapse rates and disability in the modern treatment era of neuromyelitis optica: data from a specialised UK centre. Paris: 7th joint ECTRIMS-ACTRIMS meeting, 2017.

60 Whittam D, Wilson M, Hamid S et al. What's new in neuromyelitis optica? A short review for the clinical neurologist. J Neurol 2017;264:2330-44.

Address for correspondence: Dr Saif Huda, Department of Neurology, Walton Centre NHS Foundation Trust, Liverpool L9 7LJ, UK.

Email: shuda@nhs.net 